\title{
Persuasive Evidence for Electron-Nuclear Coupling in Diluted Magnetic Colloidal Nanoplatelets Using Optically Detected Magnetic Resonance Spectroscopy
}

\author{
Rotem Strassberg, ${ }^{\dagger}$ Savas Delikanli, ${ }^{\dagger, \S, \|}$ Yahel Barak, ${ }^{\dagger, \|}$ Joanna Dehnel, ${ }^{\dagger, \|}$ Alyssa Kostadinov,
} Georgy Maikov, ${ }^{\prime}$ Pedro Ludwig Hernandez-Martinez, ${ }^{\ddagger}$ Manoj Sharma, ${ }^{\ddagger} \S_{0}$ Hilmi Volkan Demir, ${ }^{*}, \S_{\odot}$ and Efrat Lifshitz $* \dagger \oplus$

\author{
${ }^{\dagger}$ Schulich Faculty of Chemistry, Solid State Institute, Russell Berrie Nanotechnology Institute, Technion-Israel Institute of \\ Technology, Haifa 32000, Israel \\ ${ }^{\ddagger}$ Luminous Center of Excellence for Semiconductor Lighting and Displays, TPI, School of Electrical and Electronic Engineering, \\ School of Physical and Mathematical Sciences, School of Materials Science and Engineering, Nanyang Technological \\ University-NTU Singapore, 639798 Singapore \\ ${ }^{\S}$ Department of Electrical and Electronics Engineering, Department of Physics, UNAM-Institute of Materials Science and \\ Nanotechnology, Bilkent University, Ankara 06800, Turkey
}

Supporting Information

\begin{abstract}
The incorporation of magnetic impurities into semiconductor nanocrystals with size confinement promotes enhanced spin exchange interaction between photogenerated carriers and the guest spins. This interaction stimulates new magnetooptical properties with significant advantages for emerging spin-based technologies. Here we observe and elaborate on carrier-guest interactions in magnetically doped colloidal nanoplatelets with the chemical formula $\mathrm{CdSe} / \mathrm{Cd}_{1-x} \mathrm{Mn}_{x} \mathrm{~S}$, explored by optically detected magnetic resonance and magneto-photoluminescence spectroscopy. The host matrix, with a quasi-type II electronic configuration, introduces a dominant interaction between a photogenerated electron and a magnetic dopant. Furthermore, the data convincingly presents the interaction between an electron and nuclear spins of the doped ions located at neighboring surroundings, with consequent influence on the carrier's spin relaxation time. The nuclear spin contribution by the magnetic dopants in colloidal nanoplatelets is considered here for the first time.
\end{abstract}

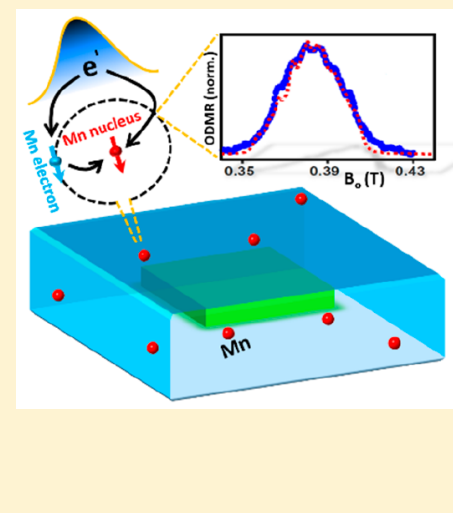

olloidal semiconductor nanocrystals have attracted widespread interest during the past three decades by virtue of their fundamental science as well as their possible use in various optoelectronic applications. ${ }^{1-3}$ In the last decade, studies paid special attention to incorporating magnetic impurities in nominally nonmagnetic colloidal semiconductors, for the formation of diluted magnetic semiconductor (DMS) nanocrystals. The interest in such nanocrystals was motivated by the ability to tailor spin properties in size-confined systems, with promising implementation in quantum information, ${ }^{4}$ spintronics, ${ }^{5}$ solar cell concentrators, ${ }^{6}$ and light-emitting diodes. $^{7}$ Past studies of bulk semiconductors have shown that doping tunes crystallographic, optical, and magentic properties. $^{8-11}$ Subsequently, the development of multiple quantum wells exposed for the first time the properties of DMS in confined systems, ${ }^{10,12}$ and naturally, DMS was also implemented in self-assembled quantum dots. ${ }^{13-18}$ However, the recent renewed attention indicates that DMSs developed in colloidal nanocrystals offer benefits beyond those found in other structures, steming from the ability to tune size confinement over a wide range, as well as from the controlled positioning of individual magnetic dopants. ${ }^{19}$ Numerous studies reported the introduction of transition-metal magnetic ions with unfilled occupancy of atomic d-orbitals into various semiconductor nanocrytals. Those DMS structures demonstrated large changes in the optical and magneto-optical properties, like giant magnetization and $g$-factors, polarization of emission recombination, or an energy transfer. ${ }^{10,19-26}$

The type of coupling between the semiconductor and dopant electronic states depends on the relative offset of their ground and first excited states. For example, the valence and conduction band edges in II-VI nanocystrals are composed from a heavy hole $\left(1 S_{\mathrm{hh}}, S=3 / 2\right)$ and an electron $\left(1 \mathrm{~S}_{\mathrm{e}}, S=1\right.$ / 2 ), respectively. A classical magnetic ion from the first row of transition metals, e.g., $\mathrm{Mn}^{2+}$ with five unpaired spins in the ground state, experiences a tetrahedral ligand field once positioned in a substitutional site in a II-VI semiconductor, thus having in turn a ground and first excited state with ${ }^{6} \mathrm{~A}_{1}$ and ${ }^{4} T_{1}$ symmetry. The heavy hole in the valence band has a

Received: July 10, 2019

Accepted: July 17, 2019

Published: July 17, 2019 

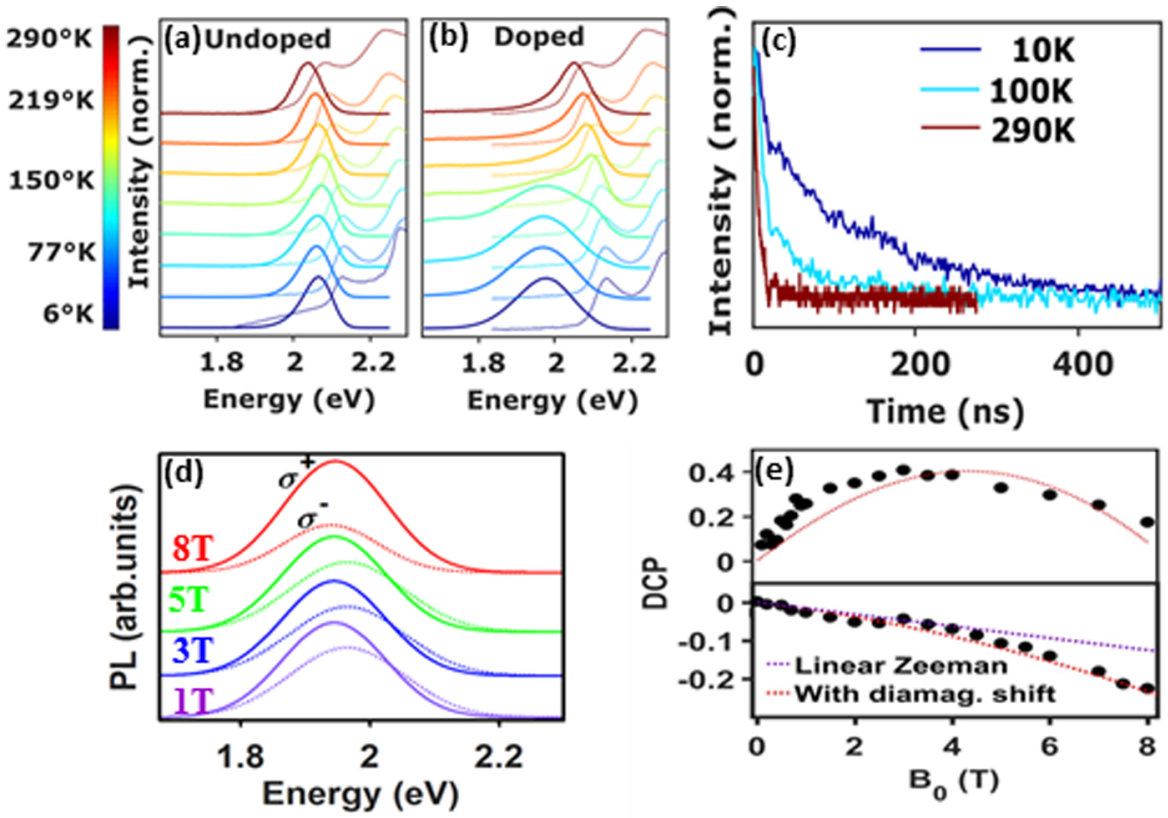

Figure 1. Optical and magneto-optical measurements: (a) absorbance curves and photoluminescence (PL) spectra of CdSe/CdS and (b) CdSe/ $\mathrm{Cd}_{1-x} \mathrm{Mn}_{x} \mathrm{~S}$ (right) NPLs recorded at various temperatures; (c) PL time-decay curve recorded at 10, 100, and $290 \mathrm{~K}$; (d) PL spectra of CdSe/ $\mathrm{Cd}_{1-x} \mathrm{Mn}_{x} \mathrm{~S}$ NPLs recorded under the influence of different magnetic field $\left(B_{0}\right)$ strength, monitored with two opposing circular polarization directions, $\left(\sigma^{ \pm}\right)$; (e) plot of the degree of circular polarization (DCP) versus strength of $B_{0}$ of $\mathrm{CdSe} / \mathrm{Cd}_{1-x} \mathrm{Mn}_{x} \mathrm{~S}$ (top panel) and CdSe/CdS (bottom panel) NPLs. The black dots are the experimental results, and the red dashed lines are fitted curves calculated according to eq 1.

minor dependence on temperature or size, thereupon, an energy offset between $1 S_{e}$ and ${ }^{4} T_{1}$ states becomes the figure of merit to determine the host-guest interaction. When ${ }^{4} \mathrm{~T}_{1}$ is situated way above $1 S_{e}$, recombination emission is dominated by the semiconductor exciton emission with typical lifetime of nanoseconds; when $1 \mathrm{~S}_{\mathrm{e}}$ is positioned way above ${ }^{4} \mathrm{~T}_{1}$, an excitation above a host band gap results in an energy transfer into guest states followed by ${ }^{4} \mathrm{~T}_{1} \rightarrow{ }^{6} \mathrm{~A}_{1}$ transition or exceptionally ${ }^{4} \mathrm{~T}_{1}$-to-conduction band repopulation, ${ }^{27}$ and both of these processes are characterized by a microsecond lifetime. When valence ( $\mathrm{p}$-orbit character) and conduction ( $\mathrm{s}$ orbit character) states are nearly in resonance with the ${ }^{6} \mathrm{~A}_{1}$ and ${ }^{4} \mathrm{~T}_{1}$ states, a mutual spin exchange process takes place, leading to the so-called $\mathrm{sp}-\mathrm{d}$ interaction, where $\mathrm{s}-\mathrm{d}$ coupling follows a potential spin exchange, while $\mathrm{p}-\mathrm{d}$ corresponds to a kinetic spin exchange process. ${ }^{28-33}$ The strength of a spin exchange is mainly governed by the degree of the electronic state offsets, as well as by the distribution function of a carrier at the site of the dopant. The $\mathrm{sp}-\mathrm{d}$ exchange induces the formation of mutual alignment of host and guest electron spins and the formation of a magnetic polaron, the last characterized by a broad emission with a lifetime exceeding $100 \mathrm{~ns}^{27}$ The $\mathrm{sp}-\mathrm{d}$ interaction is also responsible for the development of giant magnetization and variation of sign and magnitude of a carrier $g$-factor. ${ }^{28,33,34,28,35}$ The spatial confinement of the carriers within nanocrystals with diameters or thickness $<10 \mathrm{~nm}$ significantly enhances the $\mathrm{sp}-\mathrm{d}$ exchange interaction.

Previous efforts investigating magnetically doped quantum dots, ${ }^{7,19,20,22,26,33,34,36-42}$ ribbons, ${ }^{43}$ and nanoplatelets ${ }^{44-46}$ of II-VI, III-V, and I-III-V colloidal semiconductors revealed information about magnetization fluctuations, ${ }^{37,47,48}$ excitonmagnetic polaron formation, $19,37,49,50$ and giant magnetization and spin-coherence times. ${ }^{13,17,21,24,51-53}$ The studies have indicated that magnetic doping provides the colloidal semiconductors with spin degrees of freedom. Moreover, formation of core/shell heterostructures enables tunability of carrier's distribution across different parts of the heterostructure. ${ }^{54-59}$

The current work is focused on investigating magnetooptical properties of $\mathrm{CdSe} / \mathrm{CdS}$ core/shell nanoplatelets (NPLs) doped with a diluted concentration of $\mathrm{Mn}^{2+}$ ions at the shell regime. The NPLs showed thickness uniformity ${ }^{60-62}$ and narrow absorbance and emission bands. ${ }^{44,63-68}$ Further, the CdSe/CdS core/shell NPLs have a quasi-type II electronic band-edge structure, ${ }^{44}$ enabling different distribution of the carriers across the NPLs and consequently inducing selective carrier-dopant spin exchange interactions. Magneto-optical photoluminescence was used to follow the change of a $g$-factor by the incorporation of $\mathrm{Mn}^{2+}$ dopants at the shell regime. Furthermore, optically detected magnetic resonance experiments, used in the colloidal DMS research for the first time, showed persuasive evidence of the coupling between a photogenerated electron and the nuclear spins of the guest ion, appearing as a sextet manifold on top of an electron magnetic resonance band. Coupling a carrier to the dopant nuclei takes place via a direct process or, alternatively, bridging by spin exchange between the carrier and the dopant-electron. The contribution of nuclear spin coupling presumably influenced the observed spin relaxation times.

The nanoplatelets (NPLs) under investigation, undoped $\mathrm{CdSe} / \mathrm{CdS}$ and magnetically doped CdSe/ $\mathrm{Cd}_{1-x} \mathrm{Mn}_{x} \mathrm{~S}(x>0)$ core/shell heterostructures, were prepared via a procedure given in the Supporting Information. The NPLs examined consisted of a zinc-blende core-platelet with thickness/length/ width dimensions of $0.7 \mathrm{~nm} / 30 \mathrm{~nm} / 20 \mathrm{~nm}$ and a zinc-blende shell with a thickness varying from 0.35 to $1.4 \mathrm{~nm}$. A representative transmission electron microscopy (TEM) image of the NPLs examined is shown in Figure S1a. Absorbance curves of $\mathrm{CdSe}$ cores and $\mathrm{CdSe} / \mathrm{Cd}_{1-x} \mathrm{Mn}_{x} \mathrm{~S}$ core/shell NPLs recorded at room temperature are shown in panels $\mathrm{b}$ and $\mathrm{c}$ of Figure S1, respectively. The crystallographic zinc-blende 
structure was verified by X-ray diffraction, and the $\mathrm{Mn}^{2+}$ content was measured via inductively coupled plasma (ICP) analysis (1.1\%) (not shown). $\mathrm{Mn}^{2+}$ positioning within the NPLs was examined by recording a ground-state electron spin resonance (ESR) spectrum, as shown in Figure S1d (discussed below). $\mathrm{Mn}^{2+}$ has a total electron spin of $S=5 / 2$. In principle, all transitions obeying $\Delta S= \pm 1$ between the spin projections $m_{\mathrm{S}}= \pm 5 / 2, \pm 3 / 2, \pm 1 / 2$ should have been recognized in the ESR spectrum. However, most of them have strong anisotropy, and when measuring randomly oriented NPLs in a solution, the resonance lines mostly averaged out except that of $-1 / 2 \leftrightarrow$ $+1 / 2$. Thus, the ESR spectrum displays a characteristic sextet related to the $\pm 1 / 2$ of the $\mathrm{Mn}^{2+}$-electron spin transition split by a nuclear-electron hyperfine interaction (with nuclear spin $I=5 / 2)$. The hyperfine constant extracted from the ESR spectrum is $0.8 \mu \mathrm{eV}$ (6.9 mTesla), indicating the positioning of $\mathrm{Mn}^{2+}$ within the CdS crystal at the shell regime. ${ }^{69,70}$

Absorbance and photoluminescence (PL) spectra of CdSe/ $\mathrm{CdS}$ and $\mathrm{CdSe} / \mathrm{Cd}_{1-x} \mathrm{Mn}_{x} \mathrm{~S}$ NPLs (both with shell thickness of $1.4 \mathrm{~nm}$ ) recorded at various temperatures are shown Figure la,b by thin and bold lines, respectively. The absorbance curves within the displayed spectral window include the first and second exciton transitions, both shifted to lower energies with an increase of the temperature following a Varshni trend as commonly found in II-VI semiconductors. The PL spectra of the undoped NPLs follow a trend similar to their absorbance, despite a small deviation between 100 and 150 $\mathrm{K}$, which is compatible with behavior reported before in NPLs, ${ }^{71}$ and it is further explained in the Supporting Information. The PL temperature dependence of doped NPLs requires special attention: In the low-temperature range $(<130 \mathrm{~K})$ the spectra are dominated by a broad emission line Stokes shifted from the corresponding absorbance by $100-130 \mathrm{meV}$, and the emission band is accompanied by a weak tail at the low-energy side. The spectrum at $4 \mathrm{~K}$ is centered at $1.97 \mathrm{eV}$ and undergoes a mild gradual blue-shift with the increase of the temperature up to $130 \mathrm{~K}$ (an anti-Varshni behavior); around $130 \mathrm{~K}$, a second narrow PL band merges, which gradually becomes the dominant feature, and twists the trend to a Varshni behavior with further increase of the temperature. The anti-Varshni behavior was commonly found in magnetic doped nanocrystals, ${ }^{7,25}$ designating the formation of a magnetic polaron at temperatures < $130 \mathrm{~K}$; however, the Varshni behavior at $>130$ $\mathrm{K}$ suggests the occurrence of band-edge exciton recombination. Overall, the shift of the energy band gap with temperature with respect to the invariant-temperature ${ }^{4} \mathrm{~T}_{1}$ state induced a change of the emission process from a magnetic polaron to exciton recombination, according to criteria discussed above. It is important to note that the PL band at the lowest temperatures also resembles the appearance of ${ }^{4} \mathrm{~T}_{1} \rightarrow{ }^{6} \mathrm{~A}_{1}$ recombination emission; however, this atomistic event was reported previously to center around $2.2 \mathrm{eV}$ in zinc-blende structures. ${ }^{19}$ To exclude any doubt, Figure 1c compares representative PLdecay curves of doped NPLs at three different temperatures. The variables used for the biexponential fit of those curves are summarized in Table 1 in Methods, suggesting a dominant component of $7 \mathrm{~ns}$ at the highest temperature and $127 \mathrm{~ns}$ at the lowest temperature. Those time scales are different from what is expected from an atomistic ${ }^{4} \mathrm{~T}_{1} \rightarrow{ }^{6} \mathrm{~A}_{1}$ transition, which occurs after microseconds. It is worth noting that the main discussion below refers to solely the low-temperature emission; therefore, the nature of the high-temperature observations will not be elaborated any further.

The PL spectra of $\mathrm{CdSe} / \mathrm{Cd}_{1-x} \mathrm{Mn}_{x} \mathrm{~S}$ core/shell NPLs were further examined under the influence of an external magnetic field $\left(\mathrm{B}_{0}\right)$ while monitoring two opposing circular polarized $\left(\sigma^{ \pm}\right)$components at Faraday configuration ( $\mathrm{B}_{0} \| \mathrm{k}$-excitation). Representative circularly polarized spectra of doped NPLs are shown in Figure 1d recorded at $4 \mathrm{~K}$, showing a small energy gap between the components as well as a difference in their integrated intensities $\left(I_{\sigma \pm}\right)$. It is worth noting that one component $\left(\sigma^{+}\right)$mainly stays intact, while the other $\left(\sigma^{-}\right)$ exhibits a variation in energy. This is a phenomenon often found in polarized transition in semiconductors, originating from mixing the nonresponding component with neighboring states. The degree of circular polarization is defined as DCP = $\left(I_{\sigma+}-\mathrm{I}_{\sigma-}\right) /\left(\mathrm{I}_{\sigma+}+\mathrm{I}_{\sigma-}\right)$. A plot of DCP versus strength of the applied magnetic field (from 0 to $8 \mathrm{~T}$ ) is shown by the black circles in the top panel of Figure 1e. For comparison, a DCP plot of undoped NPLs is displayed in the bottom panel of Figure 1e. The observed DCP of the undoped NPLs was fitted to a relation, $P=-\left(\Delta E_{z} / 2 k_{\mathrm{B}} T\right)$, whereas that of doped NPLs reads as ${ }^{72}$

$$
P=-P_{0} \tanh \left(\frac{\Delta E_{\mathrm{Z}}}{2 k_{\mathrm{B}}\left(T+T_{0}\right)}\right)
$$

$P_{0}$ is a prefactor that considers the random orientation of NPLs in an ensemble, and $T_{0}$ defines a correction for $\mathrm{Mn}-\mathrm{Mn}$ dipole interactions. The exciton Zeeman splitting, $\Delta E_{\mathrm{Z}}$, for the undoped NPLs is given by $\Delta E_{\mathrm{Z}}=g_{\mathrm{ex}} \mu_{\mathrm{B}} B_{0}+\gamma B_{0}^{2}$, where the first term is the linear Zeeman contribution and the second term is a diamagnetic shift. The variable $\gamma$ is a diamagnetic shift coefficient, given as $\gamma=\mathrm{e}^{2}\left\langle r^{2}\right\rangle / 8 \mu$; $r$ is considered as the Bohr radius of the electron that is nearly the same as that of the exciton in II-VI semiconductors; $\mu$ is the exciton reduced mass. The fit to the $\operatorname{DPC}\left(B_{0}\right)$ plot of undoped NPLs demanded consideration of the diamagnetic shift. Then, the fit with the exciton Bohr radius of $3 \mathrm{~nm}$ concealed a value of $g_{\mathrm{ex}}$ to be +0.21 in undoped NPLs. The best fit variables are summarized in Table 2. For the doped NPLs, the expression of $\Delta E_{\mathrm{Z}}$ reads as $\Delta E_{\mathrm{Z}(\mathrm{Mn})}=g_{\mathrm{ex}} \mu_{\mathrm{B}} B_{0}+\gamma B_{0}^{2}+1 / 2 x N_{0}\left(f_{\mathrm{e}} \alpha-f_{\mathrm{h}} \beta\right)\left\langle\mathrm{S}_{0, \mathrm{z}}\right\rangle$, where the third term expresses the carrier-dopant electron spin exchange interaction including electron-dopant $\left(\mathrm{N}_{0} \alpha\right)$ and hole-dopant $\left(\mathrm{N}_{0} \beta\right)$ coupling with $N_{0}$ the number of cations per unit volume; $\mathrm{Mn}$ concentration $(x)$; the electron and hole distribution functions $\left(f_{\mathrm{e}, \mathrm{h}}\right)$ at the site of the $\mathrm{Mn}^{2+}$ ions; an average value of the $\mathrm{Mn}^{2+}$ spin projection on the laboratory axis $z\left(\left\langle\mathrm{~S}_{0, z}\right\rangle\right)$, considered as a negative term by convention. The values of $f_{\mathrm{e}}$ and $f_{\mathrm{h}}$ were derived from a simple electronic band structure model (see Figure S3) for NPLs with a quasi-type II configuration, where the electron probability density is larger at the core/shell interface with a further extent into the shell region. The values of the variables $N_{0} \alpha$ and $N_{0} \beta$ were adopted from analogous bulk compounds with a similar ligand field effect at the $\mathrm{Mn}^{2+}$ local site. ${ }^{73,74}$ The DCP plot of the doped NPLs is characterized by a nearly linear increase up to $4 \mathrm{~T}$, below which the $\operatorname{DCP}\left(B_{0}\right)$ trend can be fitted to an effective linear Zeeman term $\Delta E_{\mathrm{Z}}=g_{\text {eff }} \mu_{\mathrm{B}} B_{0}$, where $g_{\text {eff }}$ is the Landé $g$-factor of the exciton-magnetic polaron. Beyond 4 Telsa, the DCP value decreases with further increase of $B_{0}$ because of a counterbalance between the Zeeman and quadratic diamagnetic shift terms with respect to that of the $\mathrm{sp}-\mathrm{d}$ spin exchange term. The variables used for a best fit of 

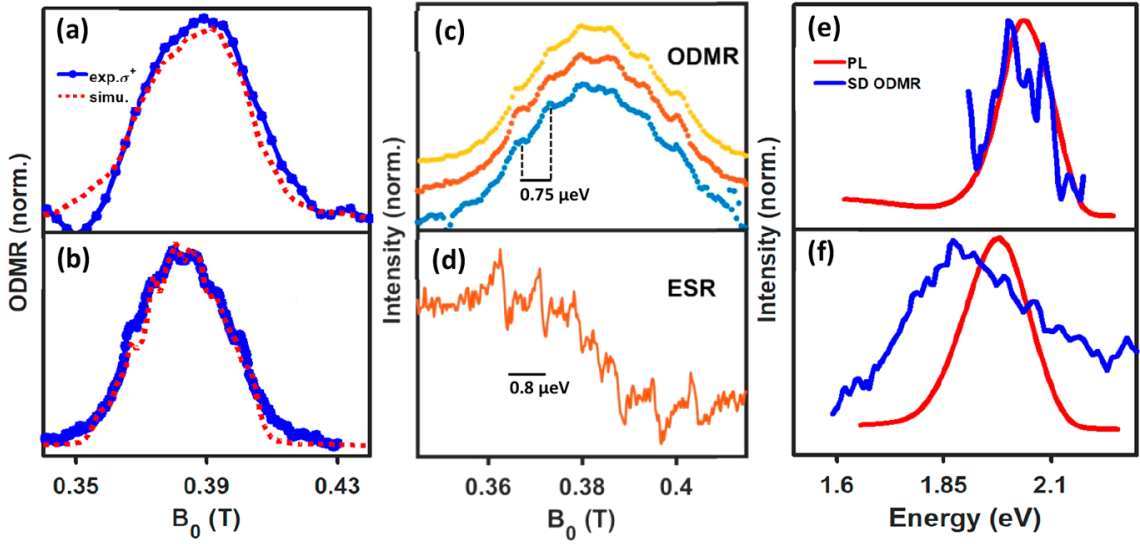

Figure 2. ODMR measurements of doped and undoped NPLs: (a and b) ODMR spectra of undoped CdSe/CdS (upper plot) and of magnetic $\mathrm{CdSe} / \mathrm{Cd}_{1-x} \mathrm{Mn}_{x} \mathrm{~S}$ (bottom plot) NPLs given by the solid blue curves, recorded under Faraday configuration and monitored with a circular polarization $\sigma^{+}$at $2.4 \mathrm{~K}$. The dashed red curves correspond to a best fit of one polarization direction, calculated using eq 2. (c) ODMR spectra similar to that shown in panel $\mathrm{b}$ recorded at various time intervals, corroborating the reproducibility of a fine structure-the sextet manifold. (d) Representative ESR spectrum of the doped NPLs of the sample shown in panel panel $b$. (e and $f$ ) Representative SD-ODMR curves of undoped (upper plot) and doped (bottom plot) NPLs (blue curves) brought together with the corresponding PL spectra (red curves).

the doped NPLs DCP curve are summarized in Table 2. It is worth mentioning that some parameters are dictated by the NPL-architecture $\left(x, N_{0}\right.$, and $\left.T_{0}\right)$ and carrier distribution $\left(f_{\mathrm{e}}\right.$ and $f_{\mathrm{h}}$ ), leaving a small degree of freedom in the simulation procedure. The best fit DCP curves are shown by the dashed red lines, revealing $g_{\text {eff }}=-4.1$ for the doped NPLs. Therefore, the giant $g$-factor in magnetically doped NPLs differs in absolute value and sign from that of the undoped NPLs. The difference is in agreement with an expected crossing of spin states as was proposed theoretically ${ }^{75}$ and in concurrence with previous studies of magnetically doped nanostructures. ${ }^{76,77}$ It is worth mentioning that dephasing effects of carrier spins by nuclear hyperfine interactions were not explicitly included in the expressions above, but they are extensively discussed below.

The optically detected magnetic resonance (ODMR) spectra were recorded when mounting NPLs in a microwave cavity at a spot of maximal attenuated magnetic field, $B_{1}$ (see a scheme in Figure S4). ${ }^{78,79}$ The cavity was installed at the center of poles of an external magnetic field, $\mathrm{B}_{0}$, to achieve magnetic resonance conditions. The NPLs were excited by a continuous light source, and magnetic resonance conditions were applied during the excited-state lifetime. The microwave source was modulated by a square-wave generator, and optical detection was synchronized by lock-in detection with the microwave modulation.

A representative ODMR spectrum of undoped $\mathrm{CdSe} / \mathrm{CdS}$ NPLs with shell thickness of $1.4 \mathrm{~nm}$ is shown by the blue curve in Figure 2a, recorded under Faraday configuration $\left(B_{0}|| k\right.$ exc) and monitored with circular polarization $\left(\sigma^{+}\right)$at $2.4 \mathrm{~K}$. The spectrum contains a single resonance band centered at $0.39 \mathrm{~T}$, slightly deviating from a symmetric Lorentzian line with a full width half-maximum of $\sim 40$ mTesla. A resonance band broadening is related to size distribution, as well as to the electron-nuclei unresolved interactions. The red curve in Figure 2a corresponds to a simulation derived by a spin Hamiltonian (see below). Observation along $\sigma^{-}$polarization showed a spectral pattern similar to that shown in Figure 2a, only slightly shifted from resonance detected at $\sigma^{+}$(not shown). The spectra recorded in Voight configuration $\left(\mathrm{B}_{0} \perp \mathrm{k}\right.$ exc) are displayed in Figure S5a. The ODMR spectra of the undoped NPLs were recorded under various powers of excitation and microwave radiation, as shown in panels $b$ and c of Figure S5, respectively. These control experiments specified the optimal conditions for ensuring excitation of a single exciton, using a laser power of $<3 \mathrm{~mW}$ and microwave intensity of $3 \mathrm{~mW}$.

A representative ODMR spectrum of doped $\mathrm{CdSe} /$ $\mathrm{Cd}_{1-x} \mathrm{Mn}_{x} \mathrm{~S}$ core/shell NPLs is shown by the blue curve in Figure $2 b$. The spectrum was recorded under Faraday configuration $\left(\mathrm{B}_{0} \| \mathrm{k}\right.$-exc) with $\sigma^{+}$polarization detection, displaying an envelope band centered at $\sim 0.38 \mathrm{~T}$, superimposed by a manifold of sextet lines, with an average intermanifold spacing of $\sim 0.75 \mu \mathrm{eV}$. Detection with $\sigma^{-}$ polarization showed a similar spectrum, which slightly shifted from the resonance detected by $\sigma^{+}$(not shown). The resonance central field of the $\sigma^{+}$detected ODMR spectrum of the doped NPLs deviates from that of the undoped NPLs by 10 mTesla, and its full width of half-maximum is narrower by $\sim 10$ mTesla. A few redundant experiments are presented in Figure 2c, displaying three different ODMR spectra of the doped NPLs recorded under the Faraday configuration at diverse times, showing consistent appearance of a sextet manifold above the wide envelope. Furthermore, Figure 2d displays an ESR spectrum of the doped NPLs with a typical hyperfine sextet. The bars marked in Figure $2 c, d$ designate the intramanifold split energy, suggesting only a slight deviation from one experiment to the other. An ODMR spectrum of $\mathrm{CdSe} / \mathrm{Cd}_{1-x} \mathrm{Mn}_{x} \mathrm{~S}$ core/shell NPLs recorded in Voight configuration is shown in Figure S5d, reflecting the linear combination spectrum of the $\sigma^{ \pm}$components.

The relation of the resonance signals in the undoped and doped NPLs to the spectral window of the corresponding PL spectra was monitored by setting a steady resonance condition and recording the PL spectra, forming the so-called spectral dependence ODMR (SD-ODMR). A steady-state condition was achieved by tuning the magnetic field to the central resonance peak and applying the microwave radiation at the operating frequency, $10.76 \mathrm{GHz}$. Representative SD-ODMR responses for $\mathrm{CdSe} / \mathrm{CdS}$ and $\mathrm{CdSe} / \mathrm{Cd}_{1-x} \mathrm{Mn}_{x} \mathrm{~S}$ NPLs are depicted by the blue curves in Figure 2e,f. The corresponding PL spectra are given for comparison by the red curves. The 

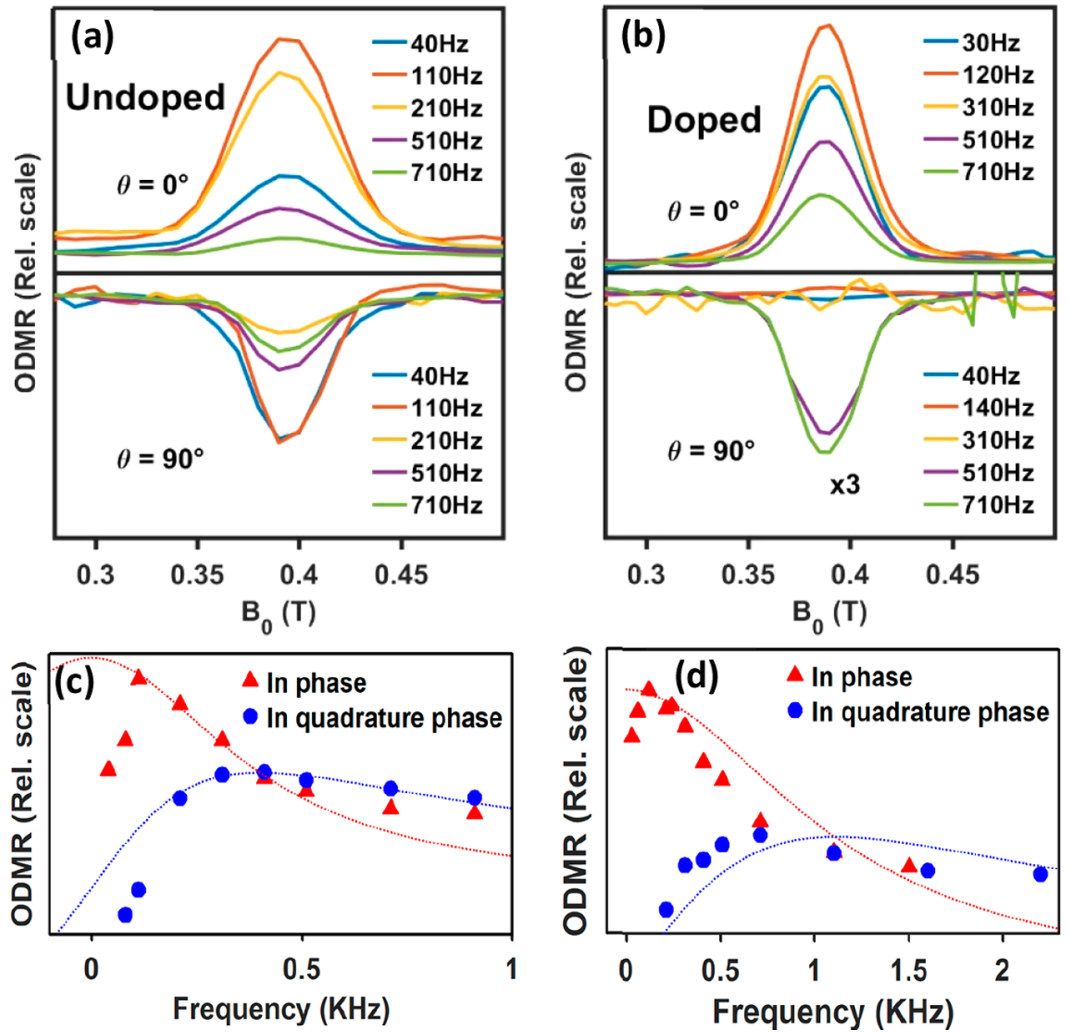

Figure 3. Following spin dynamics of carriers in NPLs: ODMR spectra of undoped (a) and doped (b) NPLs, recorded at different microwave modulation frequencies as mentioned in the insets and recorded in-phase $\left(\theta=0^{\circ}\right)$ and at quadrature phase $\left(\theta=90^{\circ}\right)$. Plots of the absolute integrated intensity of ODMR resonances versus the applied microwave modulation frequencies when measured at phase $\theta=0^{\circ}$ (red symbols) and $\theta=90^{\circ}$ (blue symbols) with respect to the microwave pulse, for the undoped (c) and doped (d) NPLs.

comparison in Figure $2 \mathrm{f}$ shows that the resonance event is related to a large part of the exciton/magnetic polaron band, with a lean toward the red side, which is more pronounced in the doped NPLs. Moreover, the SD-ODMR response of the doped NPLs covers an extra red tail in the PL spectrum, recognized as a defect band recombination. A magnetic resonance originating from two different spectral features suggests the existence of a common paramagnetic site such as an interface defect, responsible both for carrier trapping as well as exciton localization. The assignments of the paramagnetic centers are discussed below.

The ODMR methodology was further implemented to obtain spin dynamic information. Representative ODMR spectra of the undoped and doped NPLs, recorded at different microwave modulation frequencies and measured in-phase $(\theta$ $\left.=0^{\circ}\right)$ and quadrature-phase $\left(\theta=90^{\circ}\right)$ with respect to the microwave pulse, are displayed in Figure $3 \mathrm{a}, \mathrm{b}$. The spectra show that the intensity of a positive resonance is reduced gradually with the rise of the modulation frequency when measuring at $\theta=0^{\circ}$, while resonance bands appear as negative signals at $\theta=90^{\circ}$, and in any event, all measured signals preserve the circular polarization under various microwave modulation frequencies. Panels $\mathrm{c}$ and $\mathrm{d}$ of Figure 3 denote plots of absolute values of resonance intensities versus the applied microwave modulation frequencies, given by the red triangles and blue circles for the ODMR signals of undoped (Figure 3a) and doped (Figure 3b) NPLs, respectively. The red and blue dashed curves in Figure $3 \mathrm{c}, \mathrm{d}$ correspond to a fitting derived by the system frequency response described in eq 4 . The cross point between the in-phase and quadrature- phase trends provides information about the spin relaxation time. The modulation dependence ODMR spectra shown in Figure 3 are related to core/shell NPLs with shell thickness of $1.4 \mathrm{~nm}$.

The ODMR spectrum of undoped NPLs exhibited a single resonance band, associated with a spin flip of a single photogenerated carrier. Considering the band-edge composition of II-VI semiconductors (with $J_{\mathrm{h}}=3 / 2$ and $S_{\mathrm{e}}=1 / 2$ ), only the electron can undergo a direct spin flip between its spin projection component $\left(m_{\mathrm{s}}= \pm 1 / 2\right)$ by the absorption of a single microwave photon, obeying a selection rule of $\Delta S= \pm 1$. However, because of the time response of the ODMR detection (hundreds of nanoseconds up to a microsecond), direct detection of a conduction band electron (with a lifetime of a single nanosecond) cannot be achieved. Thus, the observed electron probably has larger localization with an extended lifetime as indicated by lifetime measurements (Figure 1c). According to the electron distribution calculations (Figure S3), $\sim 60 \%$ of the electron wave function is spread into the shell regime; hence, an interface localization potential acts on the electron. This localization potential can be associated with thickness fluctuations or a core/shell interface defect. Previous ODMR investigations have shown typical core/shell trapping sites such as cation or anion vacancies, created because of strain, forming twin boundaries or edge dislocations. The vacancies typically involve deficiency of neutral atoms (e.g., $\left.\mathrm{V}_{\mathrm{Cd} 0,}, \mathrm{~V}_{\mathrm{S} 0}\right)$, or ions $\left(\mathrm{V}_{\mathrm{Cd} 2+\text { or 1+ }}, \mathrm{V}_{\mathrm{S} 2 \text {-or S1- }}\right)$, where charged defects accommodate $\operatorname{spin}(\mathrm{s})$ on their own. ${ }^{78}$ It is worth noting a possible generation of a strain due to the incorporation of $\mathrm{Mn}$ ions at the core/shell interface during 

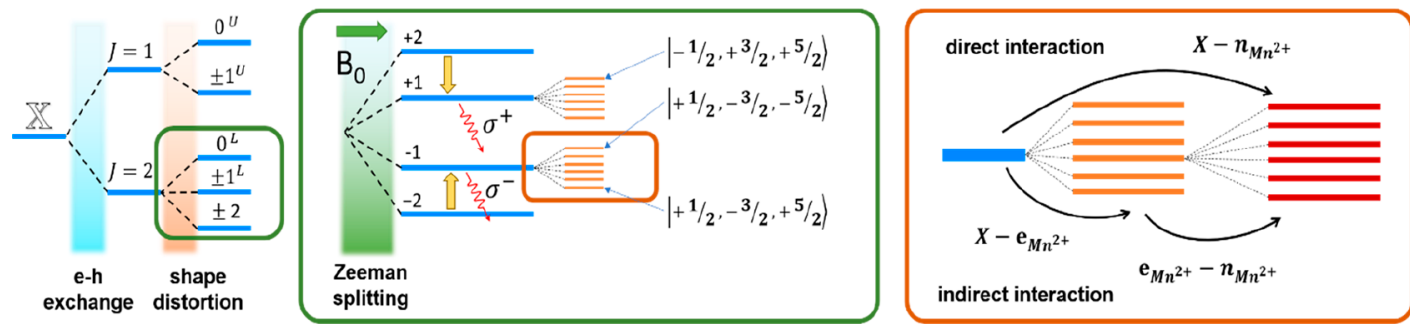

Figure 4. Diagram of the exciton and exciton-dopant electronic states. The diagram includes the following progressive interactions (from left to right): electron-hole exchange $(\mathrm{e}-\mathrm{h})$, shape or crystal distortion, Zeeman split and exciton-dopant spin exchange split. A few selective exciton$\mathrm{Mn}^{2+}$ combined states $\left|\mathrm{S}_{\mathrm{e}}\right\rangle\left|\mathrm{J}_{\mathrm{h}}\right\rangle\left|\mathrm{S}_{\mathrm{Mn}}\right\rangle$ are marked on the middle block. Coupling between a resident carrier and nuclear spins of $\mathrm{Mn}^{2+}$ ions $\left(\mathrm{e}_{\mathrm{Mn}}{ }^{2+}\right.$ $\mathrm{n}_{\mathrm{Mn}^{2+}}$ ) occurs either by direct or indirect interaction (right block). The colored horizontal lines depict the exciton (blue), Mn-electron spins (orange), and Mn-nuclear spins (red) states.

layer-by-layer deposition. As the experimental observations revealed a flip of one carrier by absorption of one microwave photon, the existence of a charged vacancy is excluded; hence, the trapping site is assumed to be electrically neutral. Neutrality is preserved in thickness fluctuations, as well as in twin boundaries accommodating both an electron and a hole in close proximity. ${ }^{80,81}$

Based on the assumption given above, the ODMR spectra of both the undoped and doped NPLs were simulated using a general spin Hamiltonian as given in eq $2:^{80,82-85}$

$$
\begin{aligned}
& \mathrm{H}_{\mathrm{s}}=\mu_{\mathrm{B}} \overrightarrow{\mathrm{S}}_{\mathrm{e}} \overline{\overline{\mathrm{g}}}_{\mathrm{e}} \overrightarrow{\mathrm{B}}_{0}+\mu_{\mathrm{B}} \overrightarrow{\mathrm{J}}_{\mathrm{h}} \overline{\overline{\mathrm{g}}}_{\mathrm{h}} \overrightarrow{\mathrm{B}}_{0}+\mu_{\mathrm{B}}\left\langle\overrightarrow{\mathrm{S}}_{\mathrm{Mn}}\right\rangle \overline{\overline{\mathrm{g}}}_{\mathrm{Mn}} \overrightarrow{\mathrm{B}}_{0} \\
& \quad+J_{\mathrm{s}-\mathrm{d}} \overrightarrow{\mathrm{S}}_{\mathrm{e}}\left\langle\overrightarrow{\mathrm{S}}_{\mathrm{Mn}}\right\rangle+J_{\mathrm{p}-\mathrm{d}} \overrightarrow{\mathrm{J}}_{\mathrm{h}}\left\langle\overrightarrow{\mathrm{S}}_{\mathrm{Mn}}\right\rangle+J_{\mathrm{e}-\mathrm{h}} \overrightarrow{\mathrm{S}}_{\mathrm{e}} \overrightarrow{\mathrm{J}}_{\mathrm{h}}+\mathrm{H}_{\mathrm{hf}}
\end{aligned}
$$

The first three terms correspond to the linear Zeeman interaction of electron $\left(S_{\mathrm{e}}\right)$, hole $\left(J_{\mathrm{h}}\right)$, and magnetic ion $\left(S_{\mathrm{Mn}}\right)$ spins with $g_{\mathrm{e}}, g_{\mathrm{h}}$, and $g_{\mathrm{Mn}} g$-factors, respectively. The host matrix, composed of II-VI semiconductors with a zinc-blende structure, determines involvement of a heavy hole with total angular momentum of $J_{\mathrm{h}}=3 / 2$ and a band-edge electron with $S_{\mathrm{e}}=1 / 2$. The ODMR resonances seen in Figure $2 \mathrm{a}$ are related to a flip of a resident electron spin when conserving spin selection rule of $\Delta S= \pm 1$, whereas a direct flip of the hole is quantum mechanically forbidden. The fourth, fifth, and sixth terms in eq 2 correspond to the $\mathrm{sp}-\mathrm{d}$ exchange interactions and isotropic electron-hole interaction, respectively, where, $J_{\mathrm{s}-\mathrm{d}}, J_{\mathrm{p}-\mathrm{d}}$ and $J_{\mathrm{e}-\mathrm{h}}$ are the electron $-\mathrm{Mn}^{2+}$, hole $-\mathrm{Mn}^{2+}$, and electron-hole exchange interactions, respectively. The electron-dopant $\left(J_{s-\mathrm{d}}\right)$ and hole-dopant $\left(J_{\mathrm{p}-\mathrm{d}}\right)$ correspond to spin exchange interactions, when $J_{s-\mathrm{d}}=x f_{\mathrm{e}} N_{0} \alpha$ and $J_{\mathrm{p}-\mathrm{d}}=$ $-x f_{\mathrm{h}} N_{0} \beta$.

The last term in eq 2 describes the hyperfine interaction, encompassing complex concerns: (1) Photogenerated carriers in Cd-based semiconductor NPLs might experience coupling to nuclear spins of rare abundance isotopes of the $\mathrm{Cd}$ atoms. Carrier depolarization due to the Overhauser effect was commonly discussed in the past in confined systems, such as multiple quantum wells and self-assembled QDs. ${ }^{86-88}$ Further, the colloidal QDs enclosed with organic molecules, i.e., hydrogen-based compounds, have already demonstrated control of spin relaxation by the hydrogen nuclei. ${ }^{36}$ The mentioned nuclei contributions cannot be excluded in the current case; however, they are not considered explicitly and instead are correlated with the overall broadening of the ODMR resonance signals. (2) The ODMR of the doped NPLs showed a pronounced sextet composition (Figure 2b) resembling the ESR spectra (Figure $2 \mathrm{~d}$ ), which deviate only by a small extent. The envelope of the resonance in Figure $2 b$ was correlated with a spin flip of a localized photogenerated electron by an interface potential, while the sextet details are correlated with the interaction between the trapped carrier and the Mn nuclear spins. Such an interaction would take place across a volume with a radius equal to that of the exciton $(\sim 3$ $\mathrm{nm})$. A simple estimation discussed in the Supporting Information approximated the existence of $\sim 3 \mathrm{Mn}$ atoms in the frame of the exciton across the core/shell interface. Likewise, previous observations on bulk semiconductors showed persuasive evidence for the increase of carrier-nuclei interactions upon localization of the photogenerated carrier. ${ }^{89}$ Accordingly, the hyperfine interaction was estimated based on an expression similar to that given in ref 86 : $\mathrm{H}_{\mathrm{hf}}=v_{0} \sum_{\mathrm{i}} A_{\mathrm{i}}^{\mathrm{I}}\left|\psi\left(\mathrm{R}_{\mathrm{i}}\right)\right|^{2} \overrightarrow{\mathrm{S}}_{\mathrm{e}}\left\langle\overrightarrow{\mathrm{I}}_{\mathrm{Mn}}^{\mathrm{i}}\right\rangle$, where $\mathrm{R}_{\mathrm{i}}$ is the position of a nucleus $\mathrm{i}$ with spin $\mathrm{I}^{\mathrm{i}}$ and with a hyperfine interaction constant $A_{\mathrm{i}}^{\mathrm{I}}$. $\mathrm{S}_{\mathrm{e}}$ and $\mathrm{I}^{\mathrm{i}}$ are the spin operators of the localized-electron and surrounding nuclei, respectively. $\nu_{0}$ is the volume of a unitary cell. Assuming a homogeneous electron wave function just across the interface at a position $\mathrm{R}_{\mathrm{i}}, \psi(\mathrm{R})$ was evaluated as $\psi(\mathrm{R})=\sqrt{2 /\left(\mathrm{v}_{0} \mathrm{~N}_{\mathrm{L}}\right)}$, with $N_{\mathrm{L}}$ being the total number of nuclei within the frame of the exciton. The effective hyperfine constant defined as $A_{\text {eff }}=v_{0} \sum_{\mathrm{i}} A_{\mathrm{i}}^{\mathrm{I}}\left|\psi\left(\mathrm{R}_{\mathrm{i}}\right)\right|^{2}$ and evaluated as $-0.5 \mu \mathrm{eV}$. The estimated $A_{\text {eff }}$ deviates slightly from that extracted from the ODMR and ESR spectra ( 0.75 and 0.80 $\mu \mathrm{eV}$, respectively). An ESR measure monitors the coupling of a $\mathrm{Mn}$ electron to its own atomic nuclei. In contrast, the ODMR measures the interaction of a resident carrier with neighboring nuclei; thus, a smaller hyperfine constant is expected. (3) Alternatively, a resident carrier is coupled to the Mn electron spin by the known $\mathrm{sp}-\mathrm{d}$ exchange mechanism; however, each Mn electron drags with it the hyperfine interaction with its own nuclei. Consequently, a ground-state hyperfine manifold is folded onto the excited-state event of a photogenerated electron spin. In other words, a resident carrier is subjected to an indirect interaction with the Mn nuclear spins, assisted by the $\mathrm{sp}-\mathrm{d}$ exchange interaction, and the given expression for $\mathrm{H}_{\mathrm{hf}}$ can be implemented also for the indirect coupling.

Based on the three arguments above, best fit curves to the ODMR signals were carried out using a spin Hamiltonian as in eq 2, which are presented by the dashed red lines in Figures $2 a, b$. The fitted curves show close agreement to the experimental results. The best fit parameters are summarized in Table 2. The electron $g$-factor has a value close to a free carrier but differs from that of a conduction band edge. ${ }^{75}$ Moreover, the electron $g$-factor is anisotropic when $g_{\mathrm{zz}}^{\mathrm{e}} \neq g_{\mathrm{xx}}^{e}=$ $g_{\mathrm{yy}}^{e}$, suggesting localization of the carrier at a distorted site, such as the core/shell interface, as proposed above and in a few 
previous publications. ${ }^{80,90,78,80}$ The proposed model agrees with the existence of an emission tail in the PL spectra of both the undoped and doped NPLs, as well as with the SD-ODMR response as shown in Figure 2e,f. Another factor, such as the electron-hole exchange interaction, exhibits values compatible with a quasi type-II heterostructures ${ }^{91}$ due to a partial carrier separation. For the magnetically doped NPLs, the values of the electron-dopant and hole-dopant interaction constants were adopted from the relations given in eq 1 .

Figure 4 displays a diagram presenting the various interactions under consideration that lead to the ODMR spectra of doped and undoped NPLs. From left to right, $\mathbb{X}$ represents an electron-hole pair consisting of a heavy hole with $J_{\mathrm{h}}=3 / 2$ and an electron with $S_{\mathrm{e}}=1 / 2$. Intrinsic electronhole exchange interaction splits $\mathbb{X}$ into a doublet $(J=2,1)$, with a further split into a 5-fold set by a crystal field and/or a shape anisotropy (left block). The presence of an external magnetic field $\left(B_{0}\right)$ lifts the remnant degeneracy (middle block). The radiative circularly polarized optical transitions are shown by the wavy red arrows. The magnetic resonance event flips an electron spin, inducing a population shift from a dark to a bright state $( \pm 2 \rightarrow \pm 1)$. This process is marked by the bold yellow arrows in the diagram. The excess density population $(\rho)$ in the bright state $| \pm 1\rangle$ is pronounced as an enhancement of luminescence intensity. Plotting the change in the luminescence intensity versus magnetic field $B_{0}$ comprised the ODMR spectrum. Further, the bright state $| \pm 1\rangle$ undergoes an additional split by a high electron spin $\mathrm{Mn}^{2+}$ ground state with $S_{M n}=5 / 2$, as marked by the orange horizontal lines. Selective combined exciton-dopant electron spin wave functions are noted next to the orange lines as $\left|S_{e}\right\rangle\left|J_{h}\right\rangle\left|S_{M n}\right\rangle$. For example, the lowest-lying bright eigenstates are $\mathrm{I}+1 /$ $2\rangle_{\mathrm{e}}|-3 / 2\rangle_{\mathrm{h}}|+5 / 2\rangle_{\mathrm{Mn}}$ and $|-1 / 2\rangle_{\mathrm{e}}|+3 / 2\rangle_{\mathrm{h}}|-5 / 2\rangle_{\mathrm{Mn}}$, and the two highest-energy states are $|-1 / 2\rangle_{\mathrm{e}}|+3 / 2\rangle_{\mathrm{h}}|+5 / 2\rangle_{\mathrm{Mn}}$ and $1+1 /$ $2\rangle_{\mathrm{e}}|-3 / 2\rangle_{\mathrm{h}}|-5 / 2\rangle_{\mathrm{Mn}}$. The character of the electron- $\mathrm{Mn}^{2+}$ exchange is ferromagnetic at the bottom of the manifold but becomes antiferromagnetic at the top. It is important to note that the dark exciton $| \pm 2\rangle$ should experience similar interaction through its coupling with $S_{M n}$ (not shown in the figure). However, the ODMR transitions obey the selection rules of $\Delta S_{\mathrm{e}}=1$ and $\Delta S_{\mathrm{Mn}}=0$. The experimental observations revealed a fine structure split of the ODMR signal, $0.75 \mu \mathrm{eV}$, a value that falls between a theoretical prediction of $10.51 \mu \mathrm{eV}$ and an ESR value of $0.8 \mu \mathrm{eV}$. This proximity unprecedentedly indicates coupling to nuclear spins with neighboring $\mathrm{Mn}^{2+}$ ions. The hyperfine split is displayed on the right block by red horizontal lines. The interaction to nuclear spins occurs either by a direct or an indirect coupling process according to the arguments above. Indirect coupling is mediated via the carrierto- $\mathrm{Mn}^{2+}$ electron coupling (the $\mathrm{sp}-\mathrm{d}$ exchange) and displayed schematically by the black arrows in the diagram (right block). Interestingly, a past report published research about an ODMR Mn-doped CdTe quantum well, depicting an extreme similarity of the resonance pattern to that of the present work, with a close hyperfine constant, ${ }^{92}$ proposing an indirect coupling of the resident carrier to the guest's nuclear spins.

A carrier-nuclear spin coupling in colloidal DMS has not been extensively discussed; however, such an interaction might have a strong influence on the magnetization and spin relaxation times. Figure 3 illustrates the dependence of the ODMR spectra on the frequency of the microwave radiation modulation. The dependence is contingent on the excess carrier density $(\rho)$ accumulated at the $| \pm 1\rangle$ following a microwave pulse, as well as on the balance between the radiative lifetime and the spin relaxation time $(\tau)$. A steadystate condition can be generated by a continuous optical excitation and a radiative lifetime comparable to the microwave "on" time. In such a case, the excess carrier density induced by microwave absorption is given by the following kinetic equation:

$$
\frac{\mathrm{d} \rho}{\mathrm{d} t}=G(1+\cos (\omega t))-\frac{\rho}{\tau}
$$

The first term in eq 3 corresponds to the microwave excitation rate; $G$ is a prefactor related to the quantum efficiency of the process, and $\omega$ is the chopping modulation frequency of the applied microwave. The second term expresses the depletion of the spin population by a spin-lattice relaxation process. Under the assumption of steady-state conditions, the following solution for the kinetic equation is proposed:

$$
\rho(t)=G \tau\left(\frac{1}{1+(\omega \tau)^{2}} \cos (\omega t)+\frac{\omega \tau}{1+(\omega \tau)^{2}} \sin (\omega t)+1\right)
$$

The first term in eq 4 is in-phase, while the second term is outof-phase (quadrature) with respect to the microwave modulation period. The third term designates a situation when $\rho(t) \geq 0$. A singular case takes place when $\tau \omega=1$, where the amplitude of the quadrature term gains a maximal value (and also bisects the in-phase curve). Therefore, this singular point enables the evaluation of the spin-relaxation time; thus, $\omega$ (quadrature $)=\omega_{\max }=1 / \tau .^{93}$

Based on the kinetic model given here, analysis of the plots depicted in Figure 3c,d revealed a carrier-dopant spin lifetime of $\sim 2.5 \mathrm{~ms}$ in undoped NPLs and $1.0 \mathrm{~ms}$ in doped NPLs of the same size and architecture. The spin relaxation of the doped NPLs was expected to be longer than that of the undoped NPLs because of mutual alignment between resident carrier and the dopant electronic spins. However, the observations showed contradictory behavior, with shorter spin relaxation in the doped NPLs. This should be related to a surplus interaction with nuclear spins, leading to decoherence and shortening of the "giant" spin lifetime. Spin decoherence via interaction with nuclear spins was widely discussed in semiconductors, governed by paramagnetic isotopes of the materials' constituents, ${ }^{86}$ and was also shown to take place in colloidal quantum dots via coupling to organic ligand nuclei. $^{94,36,95}$

In summary, the ODMR method in combination with magneto-PL spectroscopy supplied a thorough information about trapping a photogenerated electron at a core/shell interface in undoped and doped $\mathrm{CdSe} / \mathrm{CdS}$ platelets. In particular, the experiment afforded the opportunity to monitor individual carrier-dopant interactions, and the present study followed the electron-dopant coupling. The ODMR observations provided persuasive evidence for a hyperfine interaction between a localized electron at a trap interface site, with nuclear spins of next neighbor $\mathrm{Mn}^{2+}$ ions localized at the core/ shell interface. The interaction happens either directly or mediated through the carrier-to-Mn-electron spin exchange process. Coupling to nuclear spins is of paramount importance for understanding the steady-state and spin-relaxation processes in diluted magnetic nanoscale semiconductors, especially when considering those materials for emerging spin-based technologies. 


\section{METHODS}

The variables used for the biexponential fit are summarized in Table 1, and the variables used for a best fit of the doped NPLs DCP curve and for the undoped and doped NPLs ODMR spectra are summarized in Table 2.

Table 1. PL Time-Decay of Doped NPLs

$\begin{array}{crrrr}\text { temperature }(\mathrm{K}) & A_{1} & t_{1}(\mathrm{~ns}) & A_{2} & t_{2}(\mathrm{~ns}) \\ 10 & 0.3 & 9.5 & 0.7 & 127.4 \\ 20 & 0.3 & 10.8 & 0.7 & 117.8 \\ 30 & 0.3 & 7.2 & 0.7 & 103.5 \\ 50 & 0.4 & 10.7 & 0.6 & 87.0 \\ 75 & 0.6 & 11.7 & 0.4 & 84.5 \\ 100 & 0.7 & 9.5 & 0.3 & 56.8 \\ 130 & 0.9 & 7.5 & 0.1 & 31.0 \\ 160 & & 7.4 & & \\ 200 & & 7.2 & & \\ 230 & & 5.9 & & \\ 260 & & 6.7 & & \\ 290 & & 6.1 & & \end{array}$

Table 2. Physical Parameters Related to Equations 1-3

\begin{tabular}{|c|c|c|c|}
\hline parameter & value & $\begin{array}{l}\text { related } \\
\text { equation }\end{array}$ & comments \\
\hline$S$ & $5 / 2$ & \multirow[t]{8}{*}{ eqs 1 and 2} & \multirow{8}{*}{$\begin{array}{l}\text { ref } 35 \\
\text { ref } 35\end{array}$} \\
\hline$g_{\mathrm{Mn}}$ & 2.00 & & \\
\hline$x$ & $1.1 \%$ & & \\
\hline$N_{0} \alpha^{*}$ & $0.22 \mathrm{eV}$ & & \\
\hline$N_{0} \beta^{*}$ & $-1.28 \mathrm{eV}$ & & \\
\hline$f_{\mathrm{e}}$ & 0.63 & & \\
\hline$f_{\mathrm{h}}$ & 0.08 & & \\
\hline$T_{0}$ & $9 \mathrm{~K}$ & & \\
\hline$g_{\mathrm{xx}}^{\mathrm{e}}=g_{\mathrm{yy}}^{\mathrm{e}}$ & 1.90 & \multirow[t]{5}{*}{ eq 2} & \multirow{5}{*}{$\begin{array}{l}\text { fitted parameters for the ODMR } \\
\text { signal of undoped NPLs, } \\
\text { based on the spin Hamiltonian }\end{array}$} \\
\hline$g_{\mathrm{zz}}^{\mathrm{e}}$ & 2.07 & & \\
\hline$g_{\mathrm{xx}}^{\mathrm{h}}=g_{\mathrm{yy}}^{\mathrm{h}}$ & -0.86 & & \\
\hline$g_{\mathrm{zz}}^{\mathrm{h}}$ & -1.1 & & \\
\hline$J_{\mathrm{e}-\mathrm{h}}$ & $0.9 \mu \mathrm{eV}$ & & \\
\hline$g_{\mathrm{xx}}^{\mathrm{e}}=g_{\mathrm{yy}}^{\mathrm{e}}$ & 1.9 & \multirow[t]{8}{*}{ eq 2} & \multirow{10}{*}{$\begin{array}{l}\text { fitted parameters for the ODMR } \\
\text { signal of doped NPLs, based } \\
\text { on the spin Hamiltonian }\end{array}$} \\
\hline$g_{\mathrm{zz}}^{\mathrm{e}}$ & 2.1 & & \\
\hline$g_{\mathrm{xx}}^{\mathrm{h}}=\mathrm{g}_{\mathrm{yy}}^{\mathrm{h}}$ & -0.48 & & \\
\hline$g_{\mathrm{zz}}^{\mathrm{h}}$ & -0.67 & & \\
\hline $\begin{array}{c}g_{\mathrm{xx}}^{\mathrm{Mn}^{2+}}= \\
g_{\mathrm{yy}}^{\mathrm{Mn}^{2+}}= \\
g_{\mathrm{zz}}^{\mathrm{Mn}^{2+}}\end{array}$ & 2.00 & & \\
\hline$J_{s-\mathrm{d}}$ & $0.76 \mathrm{meV}$ & & \\
\hline$J_{\mathrm{p}-\mathrm{d}}$ & $-0.54 \mathrm{meV}$ & & \\
\hline$A_{\text {eff }}$ & $-0.50 \mu \mathrm{eV}$ & & \\
\hline$\tau_{\text {doped }}$ & $1 \mathrm{~ms}$ & \multirow[t]{2}{*}{ eq 3} & \\
\hline$\tau_{\text {undoped }}$ & $2.5 \mathrm{~ms}$ & & \\
\hline
\end{tabular}

\section{ASSOCIATED CONTENT}

\section{S Supporting Information}

The Supporting Information is available free of charge on the ACS Publications website at DOI: 10.1021/acs.jpclett.9b01999.

Description of the materials' preparation, microscopy, and ESR characterization; description of optical measurement setup; PL spectra of undoped NPLs; ODMR spectra in Voight configuration and as a function of light and microwave intensity; electronic band calculations and carriers' distribution functions (PDF)

\section{AUTHOR INFORMATION}

\section{Corresponding Authors}

*E-mail: ssefrat@technion.ac.il (E.F.).

*E-mail: HVDEMIR@ntu.edu.sg (H.V.D.).

ORCID $\odot$

Manoj Sharma: 0000-0001-5215-9740

Hilmi Volkan Demir: 0000-0003-1793-112X

Efrat Lifshitz: 0000-0001-7387-7821

\section{Author Contributions}

"S.D., Y.B., and J.D. contributed equally.

\section{Notes}

The authors declare no competing financial interest.

\section{ACKNOWLEDGMENTS}

E.L., R.S., Y.B., J.D., and A.K. acknowledge financial support from the Volkswagen Stiftung (No. 88116), the Israel Science Foundation (No. 914/15), the Israel Science Foundation Bikura Program (No. 1508/14), and the European Commission via the Marie-Sklodowska Curie action Phonsi (H2020MSCA-ITN-642656) and the US-Israel Binational Science Foundation (Project No.2016156). S.D., M.S., P.L.H.-M, and H.V.D. gratefully acknowledge financial support from the Singapore National Research Foundation under the program of NRF-NRFI2016-08. H.V.D. also acknowledges the support from ESF-EURYI and TUBA.

\section{REFERENCES}

(1) Brus, L. E. Electron-electron and electron-hole interactions in small semiconductor crystallites: The size dependence of the lowest excited electronic state. J. Chem. Phys. 1984, 80 (9), 4403-4409.

(2) Efros, A. L.; Efros, A. L. Interband Absorption of Light in a Semiconductor Sphere. Sov. Phys. Semicond. 1982, 16, 772.

(3) Alivisatos, A. P. Perspectives on the Physical Chemistry of Semiconductor Nanocrystals. J. Phys. Chem. 1996, 100 (31), 1322613239.

(4) DiVincenzo, D. P. Quantum Computation. Science 1995, 270 (5234), 255-261.

(5) Loss, D.; DiVincenzo, D. P. Quantum computation with quantum dots. Phys. Rev. A: At., Mol., Opt. Phys. 1998, 57 (1), 120.

(6) Chikan, V. Challenges and Prospects of Electronic Doping of Colloidal Quantum Dots: Case Study of CdSe. J. Phys. Chem. Lett. 2011, 2 (21), 2783-2789.

(7) Muckel, F.; Barrows, C. J.; Graf, A.; Schmitz, A.; Erickson, C. S.; Gamelin, D. R.; Bacher, G. Current-induced magnetic polarons in a colloidal quantum-dot device. Nano Lett. 2017, 17 (8), 4768-4773.

(8) Ohno, H. Making Nonmagnetic Semiconductors Ferromagnetic. Science 1998, 281 (5379), 951-956.

(9) Furdyna, J. K. Diluted magnetic semiconductors. J. Appl. Phys. 1988, 64 (4), R29-R64.

(10) Willardson, R. K.; Beer, A. C. In Semiconductors and semimetals; Furdyna, J. K., Kossut, J., Eds.; Academic: Boston, 1988; Vol. 25.

(11) Yoder-Short, D. R.; Debska, U.; Furdyna, J. K. Lattice parameters of $\mathrm{Zn}_{1-\mathrm{x}} \mathrm{Mn}_{\mathrm{x}} \mathrm{Se}$ and tetrahedral bond lengths in $\mathrm{A}^{\mathrm{II}}{ }_{1-\mathrm{x}} \mathrm{Mn}_{\mathrm{x}} \mathrm{B}^{\mathrm{VI}}$ alloys. J. Appl. Phys. 1985, 58 (11), 4056-4060.

(12) Jun, Y.-w.; Jung, Y.-y.; Cheon, J. Architectural Control of Magnetic Semiconductor Nanocrystals. J. Am. Chem. Soc. 2002, 124 (4), 615-619. 
(13) Oh, E.; Ramdas, A. K.; Furdyna, J. K. Magneto-optic phenomena in II-VI diluted magnetic semiconductors: the Faraday and the Voigt effect. J. Lumin. 1992, 52 (1-4), 183-191.

(14) Maksimov, A. A.; Bacher, G.; McDonald, A.; Kulakovskii, V. D.; Forchel, A.; Becker, C. R.; Landwehr, G.; Molenkamp, L. W. Magnetic polarons in a single diluted magnetic semiconductor quantum dot. Phys. Rev. B: Condens. Matter Mater. Phys. 2000, 62 (12), R7767.

(15) Lee, S.; Shin, D. Y.; Titova, L. V.; Kutrowski, M.; Furdyna, J. K.; Dobrowolska, M. Magneto-Optical Study of Multiple Layers of SelfAssembled Quantum Dots Involving Diluted Magnetic Semiconductors. J. Supercond. 2003, 16 (2), 453-456.

(16) Seufert, J.; Bacher, G.; Scheibner, M.; Forchel, A.; Lee, S.; Dobrowolska, M.; Furdyna, J. K. Dynamical Spin Response in Semimagnetic Quantum Dots. Phys. Rev. Lett. 2001, 88 (2), No. 027402.

(17) Besombes, L.; Leger, Y.; Bernos, J.; Boukari, H.; Mariette, H.; Poizat, J. P.; Clement, T.; Fernaandez-Rossier, J.; Aguado, R. Optical probing of spin fluctuations of a single paramagnetic $\mathrm{Mn}$ atom in a semiconductor quantum dot. Phys. Rev. B: Condens. Matter Mater. Phys. 2008, 78 (12), 125324.

(18) Besombes, L.; Leger, Y.; Maingault, L.; Ferrand, D.; Mariette, H.; Cibert, J. Probing the Spin State of a Single Magnetic Ion in an Individual Quantum Dot. Phys. Rev. Lett. 2004, 93 (20), 207403.

(19) Beaulac, R.; Archer, P. I.; Ochsenbein, S. T.; Gamelin, D. R. Mn2+-Doped CdSe Quantum Dots: New Inorganic Materials for Spin-Electronics and Spin-Photonics. Adv. Funct. Mater. 2008, 18 (24), 3873-3891.

(20) Norris, D. J.; Yao, N.; Charnock, F. T.; Kennedy, T. A. Highquality manganese-doped ZnSe nanocrystals. Nano Lett. 2001, 1 (1), 3-7.

(21) Awschalom, D. D.; Hong, J. M.; Chang, L. L.; Grinstein, G. Dimensional-crossover studies of magnetic susceptibility in dilutedmagnetic-semiconductor superlattices. Phys. Rev. Lett. 1987, 59 (15), 1733.

(22) Beaulac, R.; Archer, P. I.; Liu, X.; Lee, S.; Salley, G. M.; Dobrowolska, M.; Furdyna, J. K.; Gamelin, D. R. Spin-polarizable excitonic luminescence in colloidal $\mathrm{Mn} 2+$-doped CdSe quantum dots. Nano Lett. 2008, 8 (4), 1197-1201.

(23) Fernández-Rossier, J. Single-exciton spectroscopy of semimagnetic quantum dots. Phys. Rev. B: Condens. Matter Mater. Phys. 2006, 73 (4), No. 045301.

(24) Govorov, A. O.; Kalameitsev, A. V. Optical properties of a semiconductor quantum dot with a single magnetic impurity: photoinduced spin orientation. Phys. Rev. B: Condens. Matter Mater. Phys. 2005, 71 (3), No. 035338.

(25) Beaulac, R.; Schneider, L.; Archer, P. I.; Bacher, G.; Gamelin, D. R. Light-Induced Spontaneous Magnetization in Doped Colloidal Quantum Dots. Science 2009, 325 (5943), 973-976.

(26) Mendes, U. C.; Korkusinski, M.; Trojnar, A. H.; Hawrylak, P. Optical properties of charged quantum dots doped with a single magnetic impurity. Phys. Rev. B: Condens. Matter Mater. Phys. 2013, 88 (11), 115306.

(27) Beaulac, R.; Archer, P. I.; van Rijssel, J.; Meijerink, A.; Gamelin, D. R. Exciton storage by $\mathrm{Mn}^{2+}$ in colloidal $\mathrm{Mn}^{2+}$-doped CdSe quantum dots. Nano Lett. 2008, 8 (9), 2949-2953.

(28) Merkulov, I. A.; Yakovlev, D. R.; Keller, A.; Ossau, W.; Geurts, J.; Waag, A.; Landwehr, G.; Karczewski, G.; Wojtowicz, T.; Kossut, J. Kinetic exchange between the conduction band electrons and magnetic ions in quantum-confined structures. Phys. Rev. Lett. 1999, 83 (7), 1431-1434.

(29) Willardson, R. K.; Beer, A. C. Chapter in Diluted Magnetic Semiconductors. In Semiconductors and Semimetals, Vol. 25; Furdyna, J. K., Kossut, J., Eds.; Academic: London, 1988; p 413.

(30) Kośmider, K.; Fernández-Rossier, J. Electronic properties of the $\mathrm{MoS}_{2}-\mathrm{WS}_{2}$ heterojunction. Phys. Rev. B: Condens. Matter Mater. Phys. 2013, 87 (7), No. 075451.

(31) Glazov, M.; Ivchenko, E.; Besombes, L.; Léger, Y.; Maingault, L.; Mariette, H. Fine structure of exciton excited levels in a quantum dot with a magnetic ion. Phys. Rev. B: Condens. Matter Mater. Phys. 2007, 75 (20), 205313.

(32) Besombes, L.; Leger, Y.; Maingault, L.; Ferrand, D.; Mariette, H.; Cibert, J. Carrier-induced spin splitting of an individual magnetic atom embedded in a quantum dot. Phys. Rev. B: Condens. Matter Mater. Phys. 2005, 71 (16), 161307.

(33) Beaulac, R.; Feng, Y.; May, J. W.; Badaeva, E.; Gamelin, D. R.; $\mathrm{Li}, \mathrm{X}$. Orbital pathways for $\mathrm{Mn}^{2+}$-carrier sp-d exchange in diluted magnetic semiconductor quantum dots. Phys. Rev. B: Condens. Matter Mater. Phys. 2011, 84 (19), 195324.

(34) Barrows, C. J.; Vlaskin, V. A.; Gamelin, D. R. Absorption and Magnetic Circular Dichroism Analyses of Giant Zeeman Splittings in Diffusion-Doped Colloidal Cd1-xMnxSe Quantum Dots. J. Phys. Chem. Lett. 2015, 6 (15), 3076-3081.

(35) Bussian, D. A.; Crooker, S. A.; Yin, M.; Brynda, M.; Efros, A. L.; Klimov, V. I. Tunable magnetic exchange interactions in manganesedoped inverted core-shell $\mathrm{ZnSe}-\mathrm{CdSe}$ nanocrystals. Nat. Mater. 2009, 8 (1), 35-40.

(36) Schimpf, A. M.; Ochsenbein, S. T.; Gamelin, D. R. Surface Contributions to $\mathrm{Mn}^{2+}$ Spin Dynamics in Colloidal Doped Quantum Dots. J. Phys. Chem. Lett. 2015, 6 (3), 457-463.

(37) Nelson, H. D.; Bradshaw, L. R.; Barrows, C. J.; Vlaskin, V. A.; Gamelin, D. R. Picosecond Dynamics of Excitonic Magnetic Polarons in Colloidal Diffusion-Doped $\mathrm{Cd}_{1-x} \mathrm{Mn}_{x} \mathrm{Se}$ Quantum Dots. ACS Nano 2015, 9, 11177.

(38) Bradshaw, L. R.; May, J. W.; Dempsey, J. L.; Li, X.; Gamelin, D. R. Ferromagnetic excited-state $\mathrm{Mn}^{2+}$ dimers in $\mathrm{Zn}_{1-\mathrm{x}} \mathrm{Mn}_{\mathrm{x}} \mathrm{Se}$ quantum dots observed by time-resolved magnetophotoluminescence. Phys. Rev. B: Condens. Matter Mater. Phys. 2014, 89 (11), 115312.

(39) Fainblat, R.; Muckel, F.; Barrows, C. J.; Vlaskin, V. A.; Gamelin, D. R.; Bacher, G. Valence-Band Mixing Effects in the Upper-ExcitedState Magneto-Optical Responses of Colloidal $\mathrm{Mn}^{2+}$-Doped CdSe Quantum Dots. ACS Nano 2014, 8 (12), 12669-12675.

(40) Bacher, G.; Schneider, L.; Beaulac, R.; Archer, P. I.; Gamelin, D. R. Magnetic Polaron Formation Dynamics in $\mathrm{Mn}^{2+}$-Doped Colloidal Nanocrystals up to Room Temperature. J. Korean Phys. Soc. 2011, 58 (5), 1261-1266.

(41) Wolf, S. A.; Awschalom, D. D.; Buhrman, R. A.; Daughton, J. M.; von Molnar, S.; Roukes, M. L.; Chtchelkanova, A. Y.; Treger, D. M. Spintronics: A Spin-Based Electronics Vision for the Future. Science 2001, 294 (5546), 1488-1495.

(42) Awschalom, D. D.; Kikkawa, J. M. Electron Spin and Optical Coherence in Semiconductors. Phys. Today 1999, 52 (6), 33-38.

(43) Fainblat, R.; Frohleiks, J.; Muckel, F.; Yu, J. H.; Yang, J.; Hyeon, T.; Bacher, G. Quantum confinement-controlled exchange coupling in manganese (II)-doped CdSe two-dimensional quantum well nanoribbons. Nano Lett. 2012, 12 (10), 5311-5317.

(44) Delikanli, S.; Akgul, M. Z.; Murphy, J. R.; Barman, B.; Tsai, Y.; Scrace, T.; Zhang, P.; Bozok, B.; Hernández-Martínez, P. L.; Christodoulides, J.; et al. $\mathrm{Mn}^{2+}$-Doped $\mathrm{CdSe} / \mathrm{CdS}$ Core/Multishell Colloidal Quantum Wells Enabling Tunable Carrier-Dopant Exchange Interactions. ACS Nano 2015, 9 (12), 12473-12479.

(45) Murphy, J.; Delikanli, S.; Scrace, T.; Zhang, P.; Norden, T.; Thomay, T.; Cartwright, A.; Demir, H. V.; Petrou, A. Time-resolved photoluminescence study of $\mathrm{CdSe} / \mathrm{CdMnS} / \mathrm{CdS}$ core/multi-shell nanoplatelets. Appl. Phys. Lett. 2016, 108 (24), 242406.

(46) Yu, J. H.; Liu, X.; Kweon, K. E.; Joo, J.; Park, J.; Ko, K.-T.; Lee, D. W.; Shen, S.; Tivakornsasithorn, K.; Son, J. S.; et al. Giant Zeeman splitting in nucleation-controlled doped CdSe: $\mathrm{Mn}^{2+}$ quantum nanoribbons. Nat. Mater. 2010, 9 (1), 47-53.

(47) Bacher, G.; Maksimov, A. A.; Schömig, H.; Kulakovskii, V. D.; Welsch, M. K.; Forchel, A.; Dorozhkin, P. S.; Chernenko, A. V.; Lee, S.; Dobrowolska, M.; Furdyna, J. K. Monitoring Statistical Magnetic Fluctuations on the Nanometer Scale. Phys. Rev. Lett. 2002, 89 (12), 127201.

(48) Rice, W. D.; Liu, W. Y.; Baker, T. A.; Sinitsyn, N. A.; Klimov, V. I.; Crooker, S. A. Revealing giant internal magnetic fields due to spin fluctuations in magnetically doped colloidal nanocrystals. Nat. Nanotechnol. 2016, 11 (2), 137-142. 
(49) Yakovlev, D. R.; Merkulov, I. A. Chapter 8: Spin and energy transfer between carriers, magnetic ions, and lattice. In Introduction to the Physics of Diluted Magnetic Semiconductors; Kossut, J., Gaj, J. A., Eds.; Springer-Verlag: Heidelberg, 2010; pp 263-304.

(50) Yakovlev, D. R.; Ossau, W. Chapter 7: Magnetic polarons. In Introduction to the Physics of Diluted Magnetic Semiconductors; Kossut, J., Gaj, J. A., Eds.; Springer-Verlag: Heidelberg, 2010; pp 221-262.

(51) Beaulac, R.; Archer, P. I.; Liu, X.; Lee, S.; Salley, G. M.; Dobrowolska, M.; Furdyna, J. K.; Gamelin, D. R. Spin-Polarizable Excitonic Luminescence in Colloidal $\mathrm{Mn}^{2+}$-Doped CdSe Quantum Dots. Nano Lett. 2008, 8 (4), 1197-1201.

(52) Besombes, L.; Léger, Y.; Maingault, L.; Ferrand, D.; Mariette, H.; Cibert, J. Probing the Spin State of a Single Magnetic Ion in an Individual Quantum Dot. Phys. Rev. Lett. 2004, 93 (20), 207403.

(53) Fernández-Rossier, J. Single-exciton spectroscopy of semimagnetic quantum dots. Phys. Rev. B: Condens. Matter Mater. Phys. 2006, 73 (4), No. 045301

(54) Kim, S.; Fisher, B.; Eisler, H.-J. r.; Bawendi, M. Type-II Quantum Dots: CdTe/CdSe(Core/Shell) and CdSe/ZnTe(Core/ Shell) Heterostructures. J. Am. Chem. Soc. 2003, 125 (38), 1146611467.

(55) Mekis, I.; Talapin, D. V.; Kornowski, A.; Haase, M.; Weller, H. One-Pot Synthesis of Highly Luminescent CdSe/CdS Core/Shell Nanocrystals via Organometallic and "Greener" Chemical Approaches. J. Phys. Chem. B 2003, 107 (30), 7454-7462.

(56) Scholes, G. D. Controlling the Optical Properties of Inorganic Nanoparticles. Adv. Funct. Mater. 2008, 18 (8), 1157-1172.

(57) Brumer, M.; Kigel, A.; Amirav, L.; Sashchiuk, A.; Solomesch, O.; Tessler, N.; Lifshitz, E. PbSe/PbS and $\mathrm{PbSe} / \mathrm{PbSe}_{\mathrm{x}} \mathrm{S}_{1-\mathrm{x}}$ Core/Shell Nanocrystals. Adv. Funct. Mater. 2005, 15 (7), 1111-1116.

(58) Li, L.; Daou, T. J.; Texier, I.; Kim Chi, T. T.; Liem, N. Q.; Reiss, P. Highly Luminescent $\mathrm{CuInS}_{2} / \mathrm{ZnS}$ Core/Shell Nanocrystals: Cadmium-Free Quantum Dots for In Vivo Imaging. Chem. Mater. 2009, 21 (12), 2422-2429.

(59) Ivanov, S. A.; Piryatinski, A.; Nanda, J.; Tretiak, S.; Zavadil, K. R.; Wallace, W. O.; Werder, D.; Klimov, V. I. Type-II Core/Shell CdS/ZnSe Nanocrystals: Synthesis, Electronic Structures, and Spectroscopic Properties. J. Am. Chem. Soc. 2007, 129 (38), $11708-11719$.

(60) Mahler, B.; Nadal, B.; Bouet, C.; Patriarche, G.; Dubertret, B. Core/Shell Colloidal Semiconductor Nanoplatelets. J. Am. Chem. Soc. 2012, 134 (45), 18591-18598.

(61) Ithurria, S.; Tessier, M. D.; Mahler, B.; Lobo, R. P. S. M.; Dubertret, B.; Efros, A. L. Colloidal nanoplatelets with twodimensional electronic structure. Nat. Mater. 2011, 10 (12), 936941.

(62) Pedetti, S.; Ithurria, S.; Heuclin, H.; Patriarche, G.; Dubertret, B. Type-II CdSe/CdTe Core/Crown Semiconductor Nanoplatelets. J. Am. Chem. Soc. 2014, 136 (46), 16430-16438.

(63) Benchamekh, R.; Gippius, N. A.; Even, J.; Nestoklon, M. O.; Jancu, J. M.; Ithurria, S.; Dubertret, B.; Efros, A. L.; Voisin, P. Tightbinding calculations of image-charge effects in colloidal nanoscale platelets of CdSe. Phys. Rev. B: Condens. Matter Mater. Phys. 2014, 89 (3), No. 035307.

(64) Biadala, L.; Liu, F.; Tessier, M. D.; Yakovlev, D. R.; Dubertret, B.; Bayer, M. Recombination Dynamics of Band Edge Excitons in Quasi-Two-Dimensional CdSe Nanoplatelets. Nano Lett. 2014, 14 (3), 1134-1139.

(65) Lhuillier, E.; Pedetti, S.; Ithurria, S.; Nadal, B.; Heuclin, H.; Dubertret, B. Two-Dimensional Colloidal Metal Chalcogenides Semiconductors: Synthesis, Spectroscopy, and Applications. Acc. Chem. Res. 2015, 48 (1), 22-30.

(66) Tessier, M. D.; Spinicelli, P.; Dupont, D.; Patriarche, G.; Ithurria, S.; Dubertret, B. Efficient Exciton Concentrators Built from Colloidal Core/Crown CdSe/CdS Semiconductor Nanoplatelets. Nano Lett. 2014, 14 (1), 207-213.

(67) Sigle, D. O.; Hugall, J. T.; Ithurria, S.; Dubertret, B.; Baumberg, J. J. Probing Confined Phonon Modes in Individual CdSe
Nanoplatelets Using Surface-Enhanced Raman Scattering. Phys. Rev. Lett. 2014, 113 (8), No. 087402.

(68) Delikanli, S.; Guzelturk, B.; Hernández-Martínez, P. L.; Erdem, T.; Kelestemur, Y.; Olutas, M.; Akgul, M. Z.; Demir, H. V. Continuously Tunable Emission in Inverted Type-I CdS/CdSe Core/Crown Semiconductor Nanoplatelets. Adv. Funct. Mater. 2015, 25 (27), 4282-4289.

(69) Wang, S.; Jarrett, B. R.; Kauzlarich, S. M.; Louie, A. Y. Core/ shell quantum dots with high relaxivity and photoluminescence for multimodality imaging. J. Am. Chem. Soc. 2007, 129 (13), 3848-3856.

(70) Nag, A.; Sapra, S.; Nagamani, C.; Sharma, A.; Pradhan, N.; Bhat, S. V.; Sarma, D. D. A Study of Mn2+ Doping in CdS Nanocrystals. Chem. Mater. 2007, 19, 3252.

(71) Tessier, M. D.; Mahler, B.; Nadal, B.; Heuclin, H.; Pedetti, S.; Dubertret, B. Spectroscopy of Colloidal Semiconductor Core/Shell Nanoplatelets with High Quantum Yield. Nano Lett. 2013, 13 (7), $3321-3328$.

(72) Long, G.; Barman, B.; Delikanli, S.; Tsung Tsai, Y.; Zhang, P.; Petrou, A.; Zeng, H. Carrier-dopant exchange interactions in $\mathrm{Mn}$ doped $\mathrm{PbS}$ colloidal quantum dots. Appl. Phys. Lett. 2012, 101 (6), No. 062410.

(73) Jang, Y.; Shapiro, A.; Isarov, M.; Rubin-Brusilovski, A.; Safran, A.; Budniak, A. K.; Horani, F.; Dehnel, J.; Sashchiuk, A.; Lifshitz, E. Interface control of electronic and optical properties in IV-VI and IIVI core/shell colloidal quantum dots: a review. Chem. Commun. 2017, 53, 1002 .

(74) Vaxenburg, R.; Lifshitz, E. Alloy and heterostructure architectures as promising tools for controlling electronic properties of semiconductor quantum dots. Phys. Rev. B: Condens. Matter Mater. Phys. 2012, 85 (7), No. 075304.

(75) Chang, K.; Xia, J.; Peeters, F. Magnetic field tuning of the effective $\mathrm{g}$ factor in a diluted magnetic semiconductor quantum dot. Appl. Phys. Lett. 2003, 82 (16), 2661-2663.

(76) Delikanli, S.; Akgul, M. Z.; Murphy, J. R.; Barman, B.; Tsai, Y.; Scrace, T.; Zhang, P. Y.; Bozok, B.; Hernandez-Martinez, P. L.; Christodoulides, J.; Cartwright, A. N.; Petrou, A.; Demir, H. V. Mn2+-Doped CdSe/CdS Core/Multishell Colloidal Quantum Wells Enabling Tunable Carrier-Dopant Exchange Interactions. ACS Nano 2015, 9 (12), 12473-12479.

(77) Bussian, D. A.; Crooker, S. A.; Yin, M.; Brynda, M.; Efros, A. L.; Klimov, V. I. Tunable magnetic exchange interactions in manganesedoped inverted core-shell ZnSe-CdSe nanocrystals. Nat. Mater. 2009, 8 (1), 35-40.

(78) Lifshitz, E.; Fradkin, L.; Glozman, A.; Langof, L. Optically detected magnetic resonance studies of colloidal semiconductor nanocrystals. Annu. Rev. Phys. Chem. 2004, 55 (1), 509-557.

(79) Langof, L.; Ehrenfreund, E.; Lifshitz, E.; Micic, O. I.; Nozik, A. J. Continuous-Wave and Time-Resolved Optically Detected Magnetic Resonance Studies of Nonetched/Etched InP Nanocrystals. J. Phys. Chem. B 2002, 106 (7), 1606-1612.

(80) Lifshitz, E.; Porteanu, H.; Glozman, A.; Weller, H.; Pflughoefft, M.; Echymuller, A. Optically Detected Magnetic Resonance Study of CdS/HgS/CdS Quantum Dot Quantum Wells. J. Phys. Chem. B 1999, 103 (33), 6870-6875.

(81) Porteanu, H. E.; Lifshitz, E.; Pflughoefft, M.; Eychmuller, A.; Weller, H. Optical properties of $\mathrm{CdS} / \mathrm{HgS} / \mathrm{CdS}$ quantum dotquantum well structures. Phys. Status Solidi B 2001, 226 (1), 219232.

(82) Lifshitz, E.; Glozman, A.; Litvin, I. D.; Porteanu, H. Optically Detected Magnetic Resonance Studies of the Surface/Interface Properties of II-VI Semiconductor Quantum Dots. J. Phys. Chem. B 2000, 104 (45), 10449-10461.

(83) Fernandez-Rossier, J. Single-exciton spectroscopy of semimagnetic quantum dots. Phys. Rev. B: Condens. Matter Mater. Phys. 2006, 73 (4), No. 045301.

(84) Langof, L.; Fradkin, L.; Ehrenfreund, E.; Lifshitz, E.; Micic, O. I.; Nozik, A. J. Colloidal InP/ZnS core-shell nanocrystals studied by linearly and circularly polarized photoluminescence. Chem. Phys. 2004, 297 (1-3), 93-98. 
(85) Glozman, A.; Lifshitz, E.; Hoppe, K.; Rogach, A. L.; Weller, H.; Echymüller, A. Optically detected magnetic resonance of thiol-capped CdTe nanocrystals. Isr. J. Chem. 2001, 41 (1), 39-44.

(86) Le Gall, C.; Brunetti, A.; Boukari, H.; Besombes, L. Electronnuclei spin dynamics in II-VI semiconductor quantum dots. Phys. Rev. B: Condens. Matter Mater. Phys. 2012, 85 (19), 195312.

(87) Cherbunin, R. V.; Verbin, S. Y.; Auer, T.; Yakovlev, D. R.; Reuter, D.; Wieck, A. D.; Gerlovin, I. Y.; Ignatiev, I. V.; Vishnevsky, D. V.; Bayer, M. Dynamics of the nuclear spin polarization by optically oriented electrons in a (In,Ga)As/GaAs quantum dot ensemble. Phys. Rev. B: Condens. Matter Mater. Phys. 2009, 80, No. 035326.

(88) Skiba-Szymanska, J.; Chekhovich, E. A.; Nikolaenko, A. E.; Tartakovskii, A. I.; Makhonin, M. N.; Drouzas, I.; Skolnick, M. S.; Krysa, A. B. Overhauser effect in individual InP/GaxIn1-xP dots. Phys. Rev. B: Condens. Matter Mater. Phys. 2008, 77 (16), 165338.

(89) Meier, F.; Zakharchenia, B. P. Optical orientation; Elsevier Science Pub. Co.: Amsterdam, 1984.

(90) Fradkin, L.; Langof, L.; Lifshitz, E.; Rogach, A.; Gaponik, N.; Weller, H.; Eychmuller, A. Magneto-Optical Studies of $\mathrm{HgTe} /$ $\mathrm{Hg}_{\mathrm{x}} \mathrm{Cd}_{1-\mathrm{x}} \mathrm{Te}(\mathrm{S})$ Core-Shell Nanocrystals. ChemPhysChem 2003, 4 (11), 1203-1210.

(91) Cirloganu, C. M.; Padilha, L. A.; Lin, Q.; Makarov, N. S.; Velizhanin, K. A.; Luo, H.; Robel, I.; Pietryga, J. M.; Klimov, V. I. Enhanced carrier multiplication in engineered quasi-type-II quantum dots. Nat. Commun. 2014, 5, 4148.

(92) Sadowski, M. L.; Byszewski, M.; Potemski, M.; Sachrajda, A.; Karczewski, G. Optical detection of electron paramagnetic resonance in CdMnTe single quantum wells. Appl. Phys. Lett. 2003, 82 (21), 3719-3721.

(93) Epshtein, O.; Nakhmanovich, G.; Eichen, Y.; Ehrenfreund, E. Dispersive dynamics of photoexcitations in conjugated polymers measured by photomodulation spectroscopy. Phys. Rev. B: Condens. Matter Mater. Phys. 2001, 63 (12), 125206.

(94) Moro, F.; Turyanska, L.; Wilman, J.; Fielding, A. J.; Fay, M. W.; Granwehr, J.; Patane, A. Electron spin coherence near room temperature in magnetic quantum dots. Sci. Rep. 2015, 5, 10855.

(95) Ochsenbein, S. T.; Gamelin, D. R. Quantum oscillations in magnetically doped colloidal nanocrystals. Nat. Nanotechnol. 2011, 6 (2), 112-114. 